\title{
Moments of Superellipsoids and their Application to Range Image Registration
}

\author{
Aleš Jaklič, Member, IEEE, and Franc Solina, Senior Member, IEEE
}

\begin{abstract}
Cartesian moments are frequently used global geometrical features in computer vision for object pose estimation and recognition. In the paper we derive a closed form expression for 3D Cartesian moment of order $p+q+r$ of a superellipsoid in its canonical coordinate system. We also show how 3D Cartesian moment of a globally deformed superellipsoid in general position and orientation can be computed as a linear combination of 3D Cartesian moments of the corresponding non-deformed superellipsoid in canonical coordinate system. Additionally, moments of objects that are compositions of superellipsoids can be computed as simple sums of moments of individual parts.

To demonstrate practical application of the derived results we register pairs of range images based on moments of recovered compositions of superellipsoids. We use a standard technique to find centers of gravity and principal axes in pairs of range images while third-order moments are used to resolve the fourway ambiguity. Experimental results show expected improvement of recovered rigid transformation based on moments of recovered superellipsoids as compared to the registration based on moments of raw range image data. Beside object pose estimation the presented results can be directly used for object recognition with moments and/or moment invariants as object features.
\end{abstract}

Index Terms-3D Cartesian moments, superellipse, superellipsoid, transformations of 3D moments, registration

\section{INTRODUCTION}

$\mathbf{M}$ OMENT-BASED techniques have a well established tradition in object recognition and pose estimation [1]. Initial two-dimensional moment invariants techniques were extended to three-dimensions [2]-[4] and three-dimensional moments were used for object-recognition [5].

Although algorithms and methods for segmentation and recovery of superellipsoids exist (see survey in [6]), momentbased methods have not been applied to such representations. Numerical integration was proposed to compute volume and moments of inertia for superellipsoids [7]. However, numerical integration must be performed for each pair of values of shape parameters $\epsilon_{1}$ and $\epsilon_{3}$ as well as for each order of moment. Closed form expressions for computation of moments would thus allow computationally efficient application of momentbased techniques to objects represented as compositions of superellipsoids.

Recovery of superellipsoids from a single view range image is an under-constrained problem and even additional constraint

This work was supported by the Ministry of Education, Science and Sports, Republic of Slovenia (Program Computer Vision 1539-506).

The authors are with the Computer Vision Laboratory, Faculty of Computer and Information Science, University of Ljubljana, Tržaška cesta 25, 1001 Ljubljana, Slovenia (e-mail: ales.jaklic@fri.uni-lj.si; franc.solina@fri.unilj.si). of minimal volume [8] does not guarantee a precise model for a single superellipsoid like object [9]. In order to obtain a precise model several range images taken from different viewpoints have to be combined into a single data set. Many registration and range data fusion algorithms are based on some form of local minimization and require a good initial estimate of the transformation [10]-[13]. The moment based method presented in this paper could provide such an estimate.

The paper is organized as follows. In Section II we derive moments of superellipses and based on this result moments of superellipsoids in their respective canonical coordinate systems. Section III presents derivation of transformations of moments of rigidly transformed and/or globally deformed objects. These results are used to compute moments of globally deformed superellipsoids in general position and orientation. Computation of moments of compositions of volumetric parts is addressed in Section IV. Sections V and VI present the registration algorithm based on moments and the experimental results, respectively.

\section{Moments of Superellipses AND Superellipsoids}

A superellipse is defined as a closed curve in $\mathbb{R}^{2}$ (see Fig. 1 (a)), with parameters $a, b, \epsilon$ and $\omega \in[-\pi, \pi)$

$$
\mathbf{r}(\omega) \equiv\left[\begin{array}{l}
x(\omega) \\
y(\omega)
\end{array}\right] \equiv\left[\begin{array}{l}
a(\cos \omega)^{\epsilon} \\
b(\sin \omega)^{\epsilon}
\end{array}\right]
$$

while a superellipsoid is defined as a closed surface in $\mathbb{R}^{3}$ (see Fig. 1 (b)), with parameters $a, b, c, \epsilon_{1}, \epsilon_{2}$ and $(\eta, \omega) \in$ $[-\pi / 2, \pi / 2] \times[-\pi, \pi)[14]$

$$
\mathbf{r}(\eta, \omega) \equiv\left[\begin{array}{l}
x(\eta, \omega) \\
y(\eta, \omega) \\
z(\eta, \omega)
\end{array}\right] \equiv\left[\begin{array}{l}
a(\cos \eta)^{\epsilon_{1}}(\cos \omega)^{\epsilon_{2}} \\
b(\cos \eta)^{\epsilon_{1}}(\sin \omega)^{\epsilon_{2}} \\
c(\sin \eta)^{\epsilon_{1}}
\end{array}\right]
$$

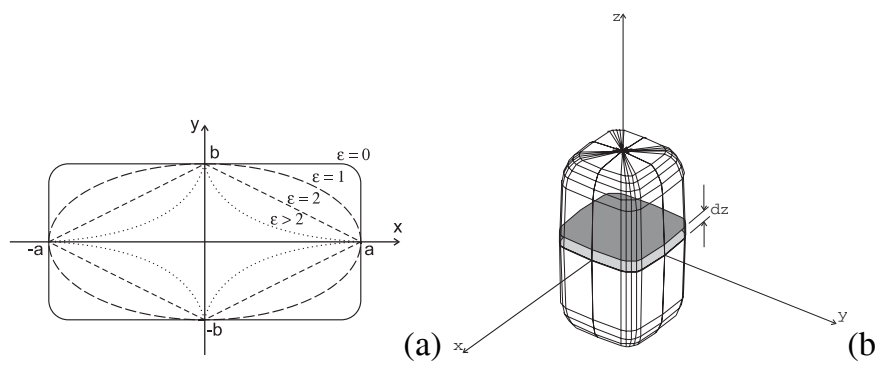

Fig. 1. (a) Superellipses for different values of parameter $\epsilon$. (b) Geometrical interpretation of a superellipsoid as a stack of superellipses with infinitesimal thickness $d z$, their size being modulated by another superellipse. 


\section{A. 2D Cartesian Moment of Order $p+q$}

The 2D Cartesian moment $m_{p q}$ of order $p+q$ of a density distribution function $f(x, y)$ is defined as a Riemann integral where $p, q=0,1,2,3, \ldots$

$$
m_{p q} \equiv \int_{-\infty}^{+\infty} \int_{-\infty}^{+\infty} x^{p} y^{q} f(x, y) d x d y \text {. }
$$

Since we are interested in solid moments of a superellipse, we set $f(x, y)=1$ inside the superellipse and $f(x, y)=0$ outside. Due to the symmetry of a superellipse with respect to $x$ and $y$ axis and the origin of the coordinate system, it is easy to note that

$$
p \text { is odd } \vee q \text { is odd } \Longrightarrow m_{p q}=0
$$

while for the case of $p$ and $q$ both being even the moment can be computed using a new coordinate system with coordinates $r$ and $\omega$ instead of $x$ and $y$. The transformation between the two systems is parameterized by $a$ and $\epsilon$ and given by

$$
\begin{aligned}
& x=\operatorname{ar}(\cos \omega)^{\epsilon} \\
& y=b r(\sin \omega)^{\epsilon}
\end{aligned}
$$

with determinant of Jacobian matrix $\mathbf{J}$ for the transformation

$$
\operatorname{det} \mathbf{J}=\left|\begin{array}{ll}
\frac{\partial x}{\partial r} & \frac{\partial x}{\partial \omega} \\
\frac{\partial y}{\partial r} & \frac{\partial y}{\partial \omega}
\end{array}\right|=\operatorname{abr} \epsilon(\sin \omega)^{\epsilon-1}(\cos \omega)^{\epsilon-1} .
$$

Since $p$ and $q$ are both even, we can reduce the computation of the integral (3) to the first quadrant of plane $x y(x \geq 0, y \geq 0)$

$$
\begin{aligned}
m_{p q} & =\int_{-\infty}^{\infty} \int_{-\infty}^{\infty} x^{p} y^{q} f(x, y) d x d y \\
& =4 \int_{0}^{\infty} \int_{0}^{\infty} x^{p} y^{q} f(x, y) d x d y \\
& =4 \int_{0}^{\pi / 2} \int_{0}^{1}\left(\operatorname{ar}(\cos \omega)^{\epsilon}\right)^{p}\left(b r(\sin \omega)^{\epsilon}\right)^{q} \operatorname{det} \mathbf{J} d r d \omega \\
& =\frac{4}{p+q+2} a^{p+1} b^{q+1} \epsilon \\
& \cdot \int_{0}^{\pi / 2}(\sin \omega)^{(q+1) \epsilon-1}(\cos \omega)^{(p+1) \epsilon-1} d \omega \\
& \frac{2}{p+q+2} a^{p+1} b^{q+1} \epsilon B\left((q+1) \frac{\epsilon}{2},(p+1) \frac{\epsilon}{2}\right)
\end{aligned}
$$

where beta function $B(x, y)$ is defined as

$$
B(x, y) \equiv 2 \int_{0}^{\pi / 2}(\sin \phi)^{2 x-1}(\cos \phi)^{2 y-1} d \phi .
$$

Table I shows the values of the derived expression for some common geometric shapes.

\section{B. 3D Cartesian Moments of Order $p+q+r$}

The 3D Cartesian moment $m_{p q r}$ of order $p+q+r$ of a density distribution function $f(x, y, z)$ is defined as Riemann integral where $p, q, r=0,1,2,3, \ldots$

$$
m_{p q r} \equiv \int_{-\infty}^{+\infty} \int_{-\infty}^{+\infty} \int_{-\infty}^{+\infty} x^{p} y^{q} z^{r} f(x, y, z) d x d y d z
$$

TABLE I

AREAS AND MOMENTS OF INERTIA FOR SUPERELLIPSES OF VARIOUS SHAPES COMPUTED FROM (7) AND USING THE LIMIT (69) FOR CASES WHERE $\epsilon=0$.

\begin{tabular}{l|c|c|c}
\hline & $\begin{array}{c}\epsilon=0 \\
\text { (rectangle) }\end{array}$ & $\begin{array}{c}\epsilon=1 \\
\text { (ellipse) }\end{array}$ & $\begin{array}{c}\epsilon=2 \\
\text { (rhomb) }\end{array}$ \\
\hline Area $\left(m_{00}\right)$ & $4 a b$ & $\pi a b$ & $2 a b$ \\
\hline $\begin{array}{l}\text { Moment of inertia about } \\
\text { the } x \text { axis }\left(m_{02}\right)\end{array}$ & $\frac{4}{3} a b^{3}$ & $\frac{\pi}{4} a b^{3}$ & $\frac{1}{3} a b^{3}$ \\
\hline $\begin{array}{l}\text { Moment of inertia about } \\
\text { the } y \text { axis }\left(m_{20}\right)\end{array}$ & $\frac{4}{3} a^{3} b$ & $\frac{\pi}{4} a^{3} b$ & $\frac{1}{3} a^{3} b$ \\
\hline
\end{tabular}

Again we set $f(x, y, z)=1$ inside the superellipsoid and $f(x, y, z)=0$ outside the superellipsoid. The moment can be expressed with a two-dimensional moment $m_{p q}$ in the plane $z=$ const. parallel to the $x y$ plane as (see Fig. 1 (b))

$$
\begin{aligned}
m_{p q r} & =\int_{-\infty}^{+\infty} z^{r}\left(\int_{-\infty}^{+\infty} \int_{-\infty}^{+\infty} x^{p} y^{q} f(x, y, z) d x d y\right) d z \\
& =\int_{-c}^{+c} z^{r} m_{p q}(z) d z
\end{aligned}
$$

Intersection of a plane parallel to $x y$ with a superellipsoid is a superellipse with parameters $a(z), b(z)$, and $\epsilon_{1}$. From (4) and the symmetry of a superellipsoid with respect to the $x y$ plane, it follows that

$$
p \text { is odd } \vee q \text { is odd } \vee r \text { is odd } \Longrightarrow m_{p q r}=0
$$

and for the case when all of $p, q$, and $r$ are even (with introduction of a new integration variable $\eta, z=c(\sin \eta)^{\epsilon_{1}}$ )

$$
\begin{aligned}
& m_{p q r}= \int_{-c}^{+c} z^{r} m_{p q}(z) d z \\
&= 2 \int_{0}^{\pi / 2} z(\eta)^{r} m_{p q}(\eta) \dot{z}(\eta) d \eta \\
&= 2 \int_{0}^{\pi / 2}\left(c(\sin \eta)^{\epsilon_{1}}\right)^{r}\left(\frac{2}{p+q+2} a^{p+1}(\eta) b^{q+1}(\eta)\right. \\
&\left.\cdot \epsilon_{2} B\left((q+1) \frac{\epsilon_{2}}{2},(p+1) \frac{\epsilon_{2}}{2}\right)\right) c \epsilon_{1}(\sin \eta)^{\epsilon_{1}-1} \cos \eta d \eta \\
&= \frac{4}{p+q+2} c^{r+1} \epsilon_{1} \epsilon_{2} B\left((q+1) \frac{\epsilon_{2}}{2},(p+1) \frac{\epsilon_{2}}{2}\right) \\
& \int_{0}^{\pi / 2}(\sin \eta)^{\epsilon_{1}(r+1)-1}\left(a(\cos \eta)^{\epsilon_{1}}\right)^{p+1}\left(b(\cos \eta)^{\epsilon_{1}}\right)^{q+1} \\
&= \frac{4}{p+q+2} a^{p+1} b^{q+1} c^{r+1} \epsilon_{1} \epsilon_{2} B\left((q+1) \frac{\epsilon_{2}}{2},(p+1) \frac{\epsilon_{2}}{2}\right) \\
& \cdot \int_{0}^{\pi / 2}(\sin \eta)^{\epsilon_{1}(r+1)-1}(\cos \eta)^{\epsilon_{1}(p+q+2)+1} d \eta \\
&= \frac{2}{p+q+2} a^{p+1} b^{q+1} c^{r+1} \epsilon_{1} \epsilon_{2} \\
& \cdot B\left((r+1) \frac{\epsilon_{1}}{2},(p+q+2) \frac{\epsilon_{1}}{2}+1\right) \\
& \epsilon_{2}\left((q+1) \frac{\epsilon_{2}}{2},(p+1) \frac{\epsilon_{2}}{2}\right) .
\end{aligned}
$$


Moments of common geometric shapes computed from (12) are presented in Table II. They correspond exactly to the well-known expressions derived by direct integration for those specific shapes [4].

\section{TABLE II}

VOLUMES AND MOMENTS OF INERTIA FOR SUPERELLIPSOIDS OF VARIOUS SHAPES COMPUTED FROM (12) AND USING LIMITS (69), (70) FOR CASES WHERE $\epsilon_{1}=0$ OR $\epsilon_{2}=0$.

\begin{tabular}{c|c|c|c}
\hline & $\begin{array}{c}\epsilon_{1}=0, \epsilon_{2}=0 \\
\text { (plate) }\end{array}$ & $\begin{array}{c}\epsilon_{1}=0, \epsilon_{2}=1 \\
\text { (elliptical cylinder) }\end{array}$ & $\begin{array}{c}\epsilon_{1}=1, \epsilon_{1}=1 \\
\text { (ellipsoid) }\end{array}$ \\
\hline$V$ & $8 a b c$ & $2 \pi a b c$ & $\frac{4}{3} \pi a b c$ \\
\hline$I_{x x}$ & $\frac{8}{3} a b c\left(b^{2}+c^{2}\right)$ & $\pi a b c\left(\frac{1}{2} b^{2}+\frac{2}{3} c^{2}\right)$ & $\frac{4}{15} \pi a b c\left(b^{2}+c^{2}\right)$ \\
\hline$I_{y y}$ & $\frac{8}{3} a b c\left(a^{2}+c^{2}\right)$ & $\pi a b c\left(\frac{1}{2} a^{2}+\frac{2}{3} c^{2}\right)$ & $\frac{4}{15} \pi a b c\left(a^{2}+c^{2}\right)$ \\
\hline$I_{z z}$ & $\frac{8}{3} a b c\left(a^{2}+b^{2}\right)$ & $\pi a b c\left(\frac{1}{2} a^{2}+\frac{1}{2} b^{2}\right)$ & $\frac{4}{15} \pi a b c\left(a^{2}+b^{2}\right)$ \\
\hline
\end{tabular}

\section{TRANSFORMATIONS OF MOMENTS}

Practical applications of superellipsoid models require their expression in arbitrary position and orientation in space as well as enhancement of their shape modeling capabilities with global deformations [6], [8], [15]. Both types of enhancements can be represented as a mapping from points of an object in coordinate system $x, y, z$ to points of a transformed objects in a new coordinate system $X, Y, Z$

$$
\begin{aligned}
X & =f_{x}(x, y, z) \\
Y & =f_{y}(x, y, z) \\
Z & =f_{z}(x, y, z) .
\end{aligned}
$$

To compute moments $M_{p q r}$ of a transformed object in coordinate system $X, Y, Z$ integration has to be performed over the volume $(V)$ bounded by the mapping of original volume $(v)$. This can be changed to integration over the volume $(v)$ bounded by original object by a change of variables in the multiple integral and using the determinant of Jacobian matrix of the mapping

$$
\operatorname{det} \mathbf{J}=\operatorname{det}\left[\begin{array}{lll}
\frac{\partial X}{\partial x} & \frac{\partial X}{\partial y} & \frac{\partial X}{\partial z} \\
\frac{\partial Y}{\partial x} & \frac{\partial Y}{\partial y} & \frac{\partial Y}{\partial z} \\
\frac{\partial Z}{\partial x} & \frac{\partial Z}{\partial y} & \frac{\partial Z}{\partial z}
\end{array}\right]
$$

The corresponding moments in the coordinate system $X, Y, Z$ and in the coordinate system $x, y, z$ are denoted as $M_{p q r}$ and $m_{p q r}$ respectively

$$
\begin{aligned}
M_{p q r} & =\iiint_{V} X^{p} Y^{q} Z^{r} d X d Y d Z \\
& =\iiint_{v} f_{x}(x, y, z)^{p} f_{y}(x, y, z)^{q} f_{z}(x, y, z)^{r}
\end{aligned}
$$$$
\cdot \operatorname{det} \mathbf{J} d x d y d z \text {. }
$$

If functions $f_{x}, f_{y}, f_{z}$ are polynomials with multiple variables $x, y, z$, the determinant of Jacobian matrix is also a polynomial of the same kind and the whole integrand in (15) can be expanded as a linear combination of moments of the original object. Alternatively, non-polynomial functions $f_{x}, f_{y}, f_{z}$ can be approximated with polynomial functions using a Taylor expansion. In the following subsections we present detailed results for translation, rotation, linear tapering and parabolic bending. Appendix II presents a program in Mathematica that can assist in derivations of expressions for a particular moment. Note that the derived results are applicable to any shape not just superellipsoids.

\section{A. Object Translation}

Translation is defined by a mapping

$$
\begin{aligned}
& f_{x}(x, y, z)=x+p_{x} \\
& f_{y}(x, y, z)=y+p_{y} \\
& f_{z}(x, y, z)=z+p_{z}
\end{aligned}
$$

where

$$
\operatorname{det} \mathbf{J}=\operatorname{det}\left[\begin{array}{lll}
1 & 0 & 0 \\
0 & 1 & 0 \\
0 & 0 & 1
\end{array}\right]=1
$$

and by using binomial theorem, it follows

$$
\begin{aligned}
M_{p q r} & =\iiint_{v}\left(x+p_{x}\right)^{p}\left(y+p_{y}\right)^{q}\left(z+p_{z}\right)^{r} d x d y d z \\
& =\sum_{i=0}^{p} \sum_{j=0}^{q} \sum_{k=0}^{r}\left(\begin{array}{l}
p \\
i
\end{array}\right)\left(\begin{array}{l}
q \\
j
\end{array}\right)\left(\begin{array}{l}
r \\
k
\end{array}\right) p_{x}^{p-i} p_{y}^{q-j} p_{z}^{r-k} m_{i j k} .
\end{aligned}
$$

Moment $M_{p q r}$ of order $n$ of a translated object is thus a linear combination of moments $m_{p q r}$ of order less or equal to $n$ of the original object.

\section{B. Object Rotation}

Rotation is defined by a mapping

$$
\begin{aligned}
& f_{x}(x, y, z)=n_{x} x+o_{x} y+a_{x} z \\
& f_{y}(x, y, z)=n_{y} x+o_{y} y+a_{y} z \\
& f_{z}(x, y, z)=n_{z} x+o_{z} y+a_{z} z
\end{aligned}
$$

where the Jacobian matrix is equal to an orthonormal rotation matrix

$$
\operatorname{det} \mathbf{J}=\operatorname{det}\left[\begin{array}{ccc}
n_{x} & o_{x} & a_{x} \\
n_{y} & o_{y} & a_{y} \\
n_{z} & o_{z} & a_{z}
\end{array}\right]=1
$$

and with the use of the multinomial theorem to expand the power terms we derive

$$
\begin{gathered}
M_{p q r}=\iiint_{v}\left(n_{x} x+o_{x} y+a_{x} z\right)^{p}\left(n_{y} x+o_{y} y+a_{y} z\right)^{q} \\
\cdot \sum_{\substack{i+j+k \\
=}} \sum_{\substack{++m+n \\
=}} \sum_{\substack{t+u+v \\
=}}(i, j, k) !(l, m, n) !(t, u, v) ! \\
\cdot n_{x}^{i} o_{x}^{j} a_{x}^{k} n_{y}^{l} o_{y}^{m} a_{y}^{n} n_{z}^{t} o_{z}^{u} a_{z}^{v} \\
\cdot m_{(i+l+t)(j+m+u)(k+n+v)}
\end{gathered}
$$

Note that moment $M_{p q r}$ of order $n$ of a rotated object is a linear combination of moments $m_{p q r}$ of the same order $n$ of the original object. 


\section{Rigid Object Transformation - Rotation and Translation}

Any rigid transformation can be decomposed into rotation followed by translation and described by

$$
\begin{aligned}
& f_{x}(x, y, z)=n_{x} x+o_{x} y+a_{x} z+p_{x} \\
& f_{y}(x, y, z)=n_{y} x+o_{y} y+a_{y} z+p_{y} \\
& f_{z}(x, y, z)=n_{z} x+o_{z} y+a_{z} z+p_{z}
\end{aligned}
$$

where

$$
\operatorname{det} \mathbf{J}=\operatorname{det}\left[\begin{array}{ccc}
n_{x} & o_{x} & a_{x} \\
n_{y} & o_{y} & a_{y} \\
n_{z} & o_{z} & a_{z}
\end{array}\right]=1
$$

A general expression for $M_{p q r}$ can be derived analogously to (21) using multinomial theorem.

$$
\begin{aligned}
& M_{p q r}=\sum_{\substack{i+j+k+l \\
=p}} \sum_{\substack{m+n+o+s \\
=q}} \sum_{\substack{t+u+v+w \\
=r}}(i, j, k, l) !(m, n, o, s) ! \\
& \cdot(t, u, v, w) ! n_{x}^{i} o_{x}^{j} a_{x}^{k} p_{x}^{l} n_{y}^{m} o_{y}^{n} a_{y}^{o} p_{y}^{s} n_{z}^{t} o_{z}^{u} a_{z}^{v} p_{z}^{w} \\
& \text { - } m_{(i+m+t)(j+n+u)(k+o+v)}
\end{aligned}
$$

where multinomial coeficients are defined as

$$
\left(n_{1}, n_{2}, \ldots, n_{k}\right) !=\frac{\left(n_{1}+n_{2}+\cdots+n_{k}\right) !}{n_{1} ! n_{2} ! \cdots n_{k} !} .
$$

However, as order of moment increases, the number of terms in polynomial expansion increases very rapidly in case of a general object. In those cases it is easier to decompose the rigid transformation into rotation followed by translation and apply two separate transformations in a sequence to the original moments. Symmetry of superellipsoids further simplifies the computation of expressions since most moments are equal to 0 in the canonical coordinate system.

\section{Linear Tapering}

Linear tapering along the $z$ axis is defined as [8]

$$
\begin{array}{ll}
f_{x}(x, y, z)=\left(\frac{k_{x}}{c} z+1\right) x & -1 \leq k_{x} \leq 1 \\
f_{y}(x, y, z)=\left(\frac{k_{y}}{c} z+1\right) y & -1 \leq k_{y} \leq 1 \\
f_{z}(x, y, z)=z &
\end{array}
$$

with

$$
\begin{aligned}
\operatorname{det} \mathbf{J} & =\operatorname{det}\left[\begin{array}{ccc}
\frac{k_{x}}{c} z+1 & 0 & \frac{k_{x}}{c} x \\
0 & \frac{k_{y}}{c} z+1 & \frac{k_{y}}{c} y \\
0 & 0 & 1
\end{array}\right] \\
& =\left(\frac{k_{x}}{c} z+1\right)\left(\frac{k_{y}}{c} z+1\right)
\end{aligned}
$$

and allows for modeling of cones and pyramids with superellipsoids. The mapping parameters $k_{x}$ and $k_{y}$ are constrained to prevent a degenerate transformation for the case of superellipsoids. Moments of a tapered superellipsoid are related to moments of a non-deformed superellipsoid as follows

$$
\begin{aligned}
M_{p q r}= & \iiint_{v}\left(\frac{k_{x}}{c} z+1\right)^{p} x^{p}\left(\frac{k_{y}}{c} z+1\right)^{q} y^{q} z^{r}\left(\frac{k_{x}}{c} z+1\right) \\
& \cdot\left(\frac{k_{y}}{c} z+1\right) d x d y d z \\
= & \iiint_{v}\left(\frac{k_{x}}{c} z+1\right)^{p+1}\left(\frac{k_{y}}{c} z+1\right)^{q+1} x^{p} y^{q} z^{r} d x d y d z \\
= & \sum_{i=0}^{p+1} \sum_{j=0}^{q+1}\left(\begin{array}{c}
p+1 \\
i
\end{array}\right)\left(\begin{array}{c}
q+1 \\
j
\end{array}\right) \frac{k_{x}^{i} k_{y}^{j}}{c^{i+j}} \\
= & \sum_{i=0}^{p+1} \sum_{j=0}^{q+1}\left(\begin{array}{c}
p+1 \\
i
\end{array}\right)\left(\begin{array}{c}
q+1 \\
j
\end{array}\right) \frac{k_{x}^{i} k_{y}^{j}}{c^{i+j}} m_{p q(r+i+j)} \cdot
\end{aligned}
$$

For illustration we use (28) to derive volume $V$, center of gravity $\left(c_{x}, c_{y}, c_{z}\right)$, and moment of inertia about the $z$ axis of a right circular cone from the moments $m_{p q r}$ of a nondeformed superellipsoid. A circular cone with radius $r$ and height $h$ can be modeled as a tapered superellipsoid, with the following parameters $a=b=r / 2, c=h / 2, \epsilon_{1}=0, \epsilon_{2}=1$ and $k_{x}=-1, k y=-1$.

$$
\begin{aligned}
V & =M_{000}=m_{000}+\frac{k_{x} k_{y}}{c^{2}} m_{002}=\frac{\pi}{3} r^{2} h \\
c_{x} & =\frac{M_{100}}{M_{000}}=0 \\
c_{y} & =\frac{M_{010}}{M_{000}}=0 \\
c_{z} & =\frac{M_{001}}{M_{000}}=\frac{\frac{k_{x}+k_{y}}{c} m_{002}}{m_{000}+\frac{k_{x} k_{y}}{c^{2}} m_{002}}=-\frac{h}{4} \\
I_{z z} & =M_{200}+M_{200}=m_{020}+\left(\frac{3 k_{x} k_{y}}{c^{2}}+\frac{3 k_{y}^{2}}{c^{2}}\right) m_{022} \\
& +\frac{k_{x} k_{y}^{3}}{c^{4}} m_{024}+m_{200}+\left(\frac{3 k_{x} k_{y}}{c^{2}}+\frac{3 k_{x}^{2}}{c^{2}}\right) m_{202} \\
& +\frac{k_{x}^{3} k_{y}}{c^{4}} m_{204}=\frac{\pi}{10} r^{4} h
\end{aligned}
$$

\section{E. Parabolic Bending}

Circular bending introduced in [8], [15] cannot be represented as a mapping with polynomial functions. However, for slight bending, it can be approximated by parabolic bending. A cross-section parallel to plane $x y$ of an object is translated in the direction of a unit vector $(\cos \alpha, \sin \alpha)$ with orientation angle $\alpha$ and magnitude proportional with $s$ to $z^{2}$

$$
\begin{aligned}
& f_{x}(x, y, z)=x+s \cos \alpha z^{2} \\
& f_{y}(x, y, z)=y+s \sin \alpha z^{2} \\
& f_{z}(x, y, z)=z
\end{aligned}
$$

where

$$
\operatorname{det} \mathbf{J}=\operatorname{det}\left[\begin{array}{ccc}
1 & 0 & 2 s \cos \alpha z \\
0 & 1 & 2 s \sin \alpha z \\
0 & 0 & 1
\end{array}\right]=1
$$


and

$$
\begin{aligned}
& M_{p q r}= \iiint_{v}\left(x+s \cos \alpha z^{2}\right)^{p}\left(y+s \sin \alpha z^{2}\right)^{q} z^{r} d x d y d z \\
&= \sum_{i=0}^{p} \sum_{j=0}^{q}\left(\begin{array}{l}
p \\
i
\end{array}\right)\left(\begin{array}{l}
q \\
j
\end{array}\right) s^{p+q-i-j} \cos ^{p-i} \alpha \sin ^{q-j} \alpha \\
& \cdot \iiint_{v} x^{i} y^{j} z^{r+2(p+q-i-j)} d x d y d z \\
&= \sum_{i=0}^{p} \sum_{j=0}^{q}\left(\begin{array}{l}
p \\
i
\end{array}\right)\left(\begin{array}{l}
q \\
j
\end{array}\right) s^{p+q-i-j} \cos ^{p-i} \alpha \sin ^{q-j} \alpha \\
& \cdot m_{i j(r+2(p+q-i-j))} .
\end{aligned}
$$

\section{F. Compositions of Transformations}

Transformations can be combined into sequences of transformations. For the case of recovering superellipsoids, the following sequence is usually used [8], [15]

$$
\text { Translate }(\text { Rotate }(B e n d(\text { Taper }(\mathbf{x})))) \text {. }
$$

In order to compute moments of such transformed shape primitives, moments of superellipsoids have to be transformed in the same sequence order.

\section{Moments of Compositions of Volumetric PARTs}

Objects can be modeled with individual volumetric parts that are glued together or as a union of their volumes which allows for penetration of parts into each other. We will discus a case of two penetrating volumetric parts $V_{1}$ and $V_{2}$ with density distribution functions $f_{1}(x, y, z)$ and $f_{2}(x, y, z)$ equal to 1 within the volumes of $V_{1}$ and $V_{2}$ and equal to 0 outside. We assume that in region $V_{1} \cap V_{2}$ the density distribution function $f(x, y, z)$ is the sum of $f_{1}$ and $f_{2}$. In other words, the value of the density function $f(x, y, z)$ is equal to the number of volumetric parts that include point $(x, y, z)$. The moment of such a composition is a sum of moments of individual parts

$$
\begin{aligned}
m_{p q r} & =\iiint_{V_{1} \cup V_{2}} x^{p} y^{q} z^{r}\left(f_{1}(x, y, z)+f_{2}(x, y, z)\right) d x d y d z \\
& =\iiint_{V_{1} \backslash\left(V_{1} \cap V_{2}\right)} x^{p} y^{q} z^{r} f_{1}(x, y, z) d x d y d z \\
& +\iiint_{V_{2} \backslash\left(V_{1} \cap V_{2}\right)} x^{p} y^{q} z^{r} f_{2}(x, y, z) d x d y d z \\
& +\iiint_{V_{1} \cap V_{2}} x^{p} y^{q} z^{r} f_{1}(x, y, z) d x d y d z \\
& +\iiint_{V_{1} \cap V_{2}} x^{p} y^{q} z^{r} f_{2}(x, y, z) d x d y d z \\
& =\iiint_{V_{1}} x^{p} y^{q} z^{r} f_{1}(x, y, z) d x d y d z \\
& +\iiint_{V_{2}} x^{p} y^{q} z^{r} f_{2}(x, y, z) d x d y d z \\
& =m_{p q r}^{V_{1}}+m_{p q r}^{V_{2}} .
\end{aligned}
$$

The result can be generalized to an arbitrary number of parts by a simple induction.

\section{RANGe IMAGe Registration}

The basic idea of range image registration based on moments is to construct a coordinate frame which is rigidly attached to the object in each image [1], [3], [5]. After constructing the two frames, we know their relationship to the global coordinate system and thus we also know the rigid transformation between the two frames, which is also the rigid transformation of the object. We will name the constructed frames the canonical frames. The canonical frame is constructed in two steps as follows [5]

1) In the first step, the global coordinate system $G$ is translated to the center of gravity of the object $\left(c_{x}, c_{y}, c_{z}\right)$ to form coordinate system $C^{\prime}$. Moments of individual superellipsoid parts $\left(m_{p q r}^{i}\right)$ are transformed to the global coordinate system $\left(M_{p q r}^{i}\right)$ and summed over the number of parts $N$ to compute the center of gravity.

$$
\begin{aligned}
& V=\sum_{i=1}^{N} M_{000}^{i} \\
& c_{x}=\frac{1}{V} \sum_{i=1}^{N} M_{100}^{i} \\
& c_{y}=\frac{1}{V} \sum_{i=1}^{N} M_{010}^{i} \\
& c_{z}=\frac{1}{V} \sum_{i=1}^{N} M_{001}^{i}
\end{aligned}
$$

First order moments of the object computed in $C^{\prime}$ are equal to 0 .

2) In the second step, the axes of coordinate system $C^{\prime}$ from the first step are rotated so that the axes are aligned along the axes of minimal and maximal moment of inertia. This rotation produces coordinate system $C^{\prime \prime}$ and the inertia matrix $\mathbf{I}^{\prime \prime}$ computed in frame $C^{\prime \prime}$ is diagonal. The direction of the axes of $C^{\prime \prime}$ correspond to the eigenvectors of the inertia matrix

$$
\begin{gathered}
\mathbf{I}^{\prime}=\left[\begin{array}{ccc}
I_{x x}^{\prime} & -I_{x y}^{\prime} & -I_{x z}^{\prime} \\
-I_{y x}^{\prime} & I_{y y}^{\prime} & -I_{y z}^{\prime} \\
-I_{z x}^{\prime} & -I_{z y}^{\prime} & I_{z z}^{\prime}
\end{array}\right] \\
I_{x x}^{\prime}=\sum_{i=1}^{N}\left(M_{020}^{\prime i}+M_{002}^{\prime i}\right) \\
I_{y y}^{\prime}=\sum_{i=1}^{N}\left(M_{200}^{\prime}{ }^{\prime}+M_{002}^{\prime i}\right) \\
I_{z z}^{\prime}=\sum_{i=1}^{N}\left(M_{200}^{\prime} i+M_{020}^{\prime i}\right) \\
I_{x y}^{\prime}=\sum_{i=1}^{N} M_{110}^{\prime i}
\end{gathered}
$$




$$
\begin{aligned}
& I_{x z}^{\prime}=\sum_{i=1}^{N} M_{101}^{\prime i} \\
& I_{y z}^{\prime}=\sum_{i=1}^{N} M_{011}^{\prime i}
\end{aligned}
$$

where moments $M_{p q r}^{\prime} i$ are computed in the $C^{\prime}$. For our work we freely selected the $x$ and the $z$ axes of $C^{\prime \prime}$ to correspond to the minimal and to the maximal moment of inertia, respectively. Since we are dealing only with right hand Cartesian coordinate frames, we uniquely determine the remaining third axis by fixing any two axes of the coordinate system.

Note, however, that the moments of inertia are invariant to rotation of the coordinate frame for $180^{\circ}$ about any of the coordinate axes or in other words, if $\mathbf{u}$ is an eigenvector of $\mathbf{I}^{\prime}$ so is the $-\mathbf{u}$. This leads to four possible orientations of the canonical coordinate frame $C^{\prime \prime}$ depicted in Fig. 2 [5]. How

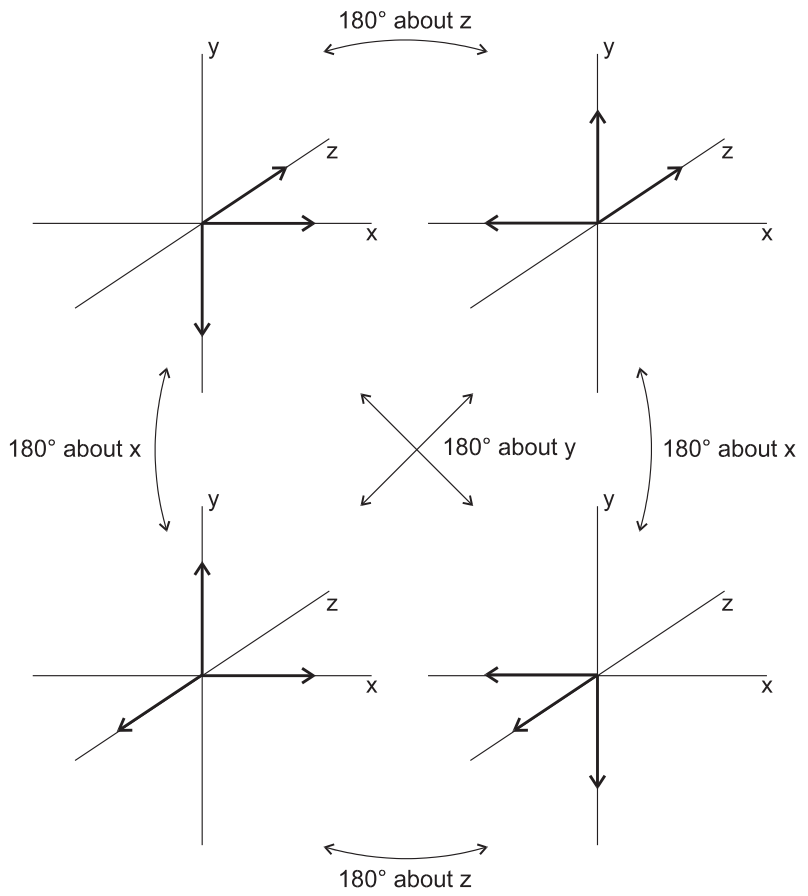

Fig. 2. Four different right hand Cartesian coordinate frames with their axes aligned along given lines. Each one of them generates the remaining three by rotations of $180^{\circ}$ about all the axes.

does this 4-way ambiguity influence image registration? In the first view we can clearly freely select one of the frames $C^{\prime \prime}$. This frame is uniquely related to spatial distribution of the object, unless the object is symmetrical. In the second view we now have four candidate frames and only one is related to the object in the second view in the same spatial way as the chosen frame in the first view. The problem is how to find this frame or the correct transformation. It is illustrated in Fig. 3.

\section{A. Resolving 4-way Ambiguity}

A search for the most distant point on the object from the origin of the coordinate system along the principal axes was proposed in [5], and the use of third order moments in [3],

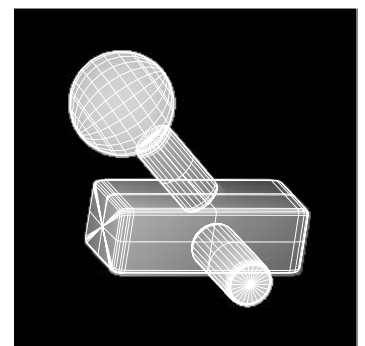

(a)

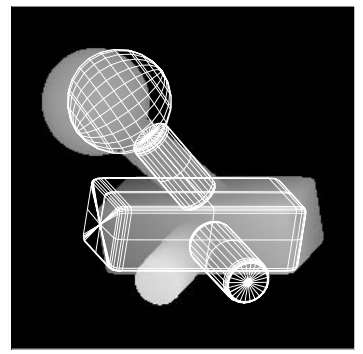

(b)
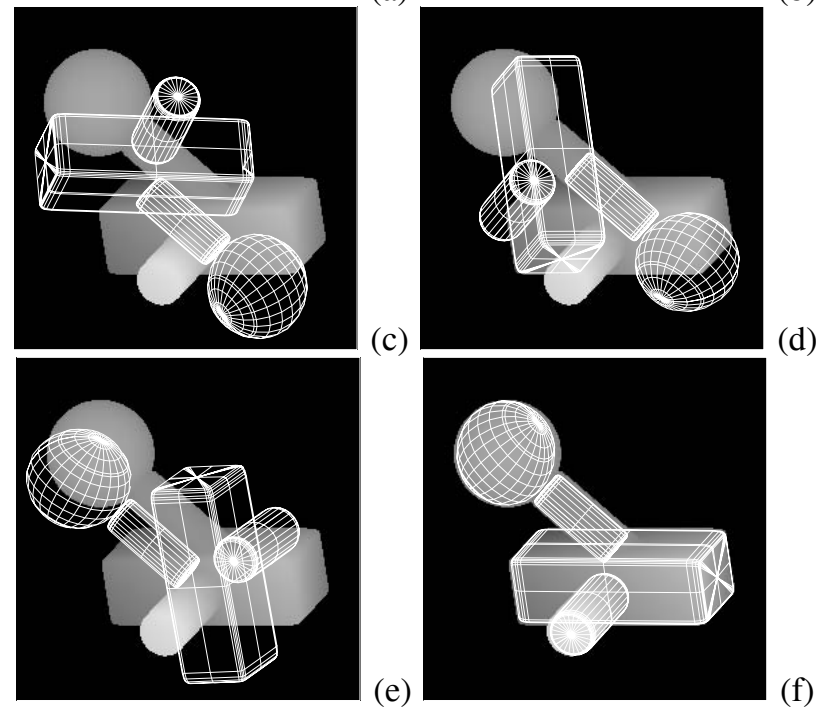

(d)

(f)

Fig. 3. Registration based on moments produces four possible solutions for the canonical frame $C^{\prime \prime}$. (a) view-1 range image and recovered model-1 (b) view-2 range image with overlaid model-1 using unit transformation $\mathbf{T}=\mathbf{I}$, figures (c), (d) (e) and (f) represent view-2 range image overlaid with model1 transformed with four possible transformations. Only the transformation depicted in figure (f) is correct.

to resolve the 4-way ambiguity. The presented approach is similar to [3], but with much simpler derivation.

It is instructive to determine how solid moments of the same object computed in the four coordinate frames are related. Let $M_{p q r}$ be a moment of an object computed in a Cartesian coordinate system, then it is easy to show that moments of the same object in the coordinate systems that are rotated for $180^{\circ}$ about $x, y$, and $z$ axes respectively are related as follows

$$
\begin{aligned}
& M_{p q r}^{\mathrm{x}}=(-1)^{q+r} M_{p q r} \\
& M_{p q r}^{\mathrm{y}}=(-1)^{p+r} M_{p q r} \\
& M_{p q r}^{\mathrm{z}}=(-1)^{p+q} M_{p q r} .
\end{aligned}
$$

We can now answer the question if moments can be used as features to resolve the 4-way ambiguity. Zeroth order moment cannot be used since it is invariant to any rigid transformation. Similarly, all first order moments computed in frames $C^{\prime \prime}$ are 0 by definition of $C^{\prime \prime}$. Second order moments $m_{110}, m_{011}, m_{101}$ are equal to 0 in frame $C^{\prime \prime}$ by definition, while $m_{200}, m_{020}, m_{002}$ are invariant to rotations that generate frames $C^{\prime \prime}$. Only 3rd and higher order moments computed in frames $C^{\prime \prime}$ provide sufficient information to distinguish frames $C^{\prime \prime}$.

We propose the following algorithm to resolve the 4-way ambiguity 
1) We select any frame from the first view and compute a vector of moments in selected frame

$$
\begin{array}{r}
\mathbf{v}=\left(m_{300}, m_{210}, m_{201}, m_{120}, m_{111},\right. \\
\left.\quad m_{102}, m_{030}, m_{021}, m_{012}, m_{003}\right)
\end{array}
$$

2) We select any frame from the second view and compute a vector of moments in selected frame

$$
\begin{array}{r}
\mathbf{v}^{\prime}=\mathbf{v}_{0}^{\prime}=( \\
m_{300}^{\prime}, m_{210}^{\prime}, m_{201}^{\prime}, m_{120}^{\prime}, m_{111}^{\prime}, \\
\left.m_{102}^{\prime}, m_{030}^{\prime}, m_{021}^{\prime}, m_{012}^{\prime}, m_{003}^{\prime}\right)
\end{array}
$$

and the remaining 3 from $\mathbf{v}^{\prime}$

$$
\begin{aligned}
\mathbf{v}_{\mathbf{x}}^{\prime}=\mathbf{v}_{1}^{\prime} & =\left(m_{300}^{\prime},-m_{210}^{\prime},-m_{201}^{\prime}, m_{120}^{\prime}, m_{111}^{\prime}\right. \\
& \left.m_{102}^{\prime},-m_{030}^{\prime},-m_{021}^{\prime},-m_{012}^{\prime},-m_{003}^{\prime}\right) \\
\mathbf{v}_{\mathbf{y}}^{\prime}=\mathbf{v}_{2}^{\prime}= & \left(-m_{300}^{\prime}, m_{210}^{\prime},-m_{201}^{\prime},-m_{120}^{\prime}, m_{111}^{\prime}\right. \\
& \left.-m_{102}^{\prime}, m_{030}^{\prime},-m_{021}^{\prime}, m_{012}^{\prime},-m_{003}^{\prime}\right) \\
\mathbf{v}_{\mathbf{z}}^{\prime}=\mathbf{v}_{3}^{\prime}= & \left(-m_{300}^{\prime},-m_{210}^{\prime}, m_{201}^{\prime},-m_{120}^{\prime}, m_{111}^{\prime},\right. \\
& \left.-m_{102}^{\prime},-m_{030}^{\prime}, m_{021}^{\prime},-m_{012}^{\prime}, m_{003}^{\prime}\right)
\end{aligned}
$$

3) The corresponding frame $i$ in the second view is the one with vector that minimizes

$$
\left\|\mathbf{v}-\mathbf{v}^{\prime}{ }_{i}\right\|
$$

Note that if third-order moments are equal to 0 due to object shape or the vector $\mathbf{v}$ is equidistant to several vectors, higher order moments may be used in the same way.

\section{EXPERIMENTAL RESULTS}

In the first experiment (Fig. 4) we recovered estimate of a rigid transformation between two range image views of a pile of stones. Algorithm described in [6], [16] was used to recover superellipsoid models from range images. The ground truth transformation $\mathbf{T}_{\text {true }}$ was computed from 7 pairs of range points corresponding to small dents visible in the gray scale images of both views. The pairs of features were manually selected. We used a least-square method described in [17] to compute $\mathbf{T}_{\text {true }}$. The estimate of transformation $\mathbf{T}_{\text {estimate }}$ was then computed based on moments of recovered superellipsoids in each view and the residual transformation $\mathbf{T}_{\text {residual }}$ was computed from (59). All transformations were represented with homogeneous transformation matrices.

$$
\mathbf{T}_{\text {true }}=\mathbf{T}_{\text {residual }} \mathbf{T}_{\text {estimate }} \text {. }
$$

A precise $\mathbf{T}_{\text {estimate }}$ would yield $\mathbf{T}_{\text {residual }}$ equal to an identity matrix. The residual transformation was decomposed into rotation followed by translation $(\mathbf{t})$. The rotation was represented by a unit vector in direction of axis of rotation (n) and an angle of rotation $(\theta)$. To visualize the quality of recovered estimate of the rigid transformation we overlaid the recovered models from view2 over the range image view1 (Fig. 4 (e)) and recovered models from view1 over the range image view2 (Fig. 4 (f)).

In the second experiment (Figs. 5-7), we generated a set of synthetic range images of an object modeled with superellipsoids to exclude errors due to non-superellipsoid shapes in object domain. Estimates of rigid transformations
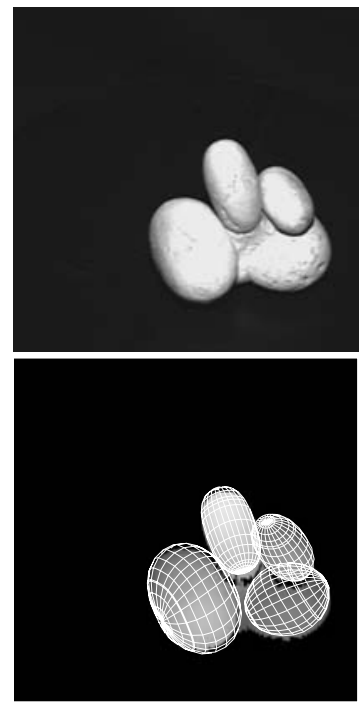

(a)
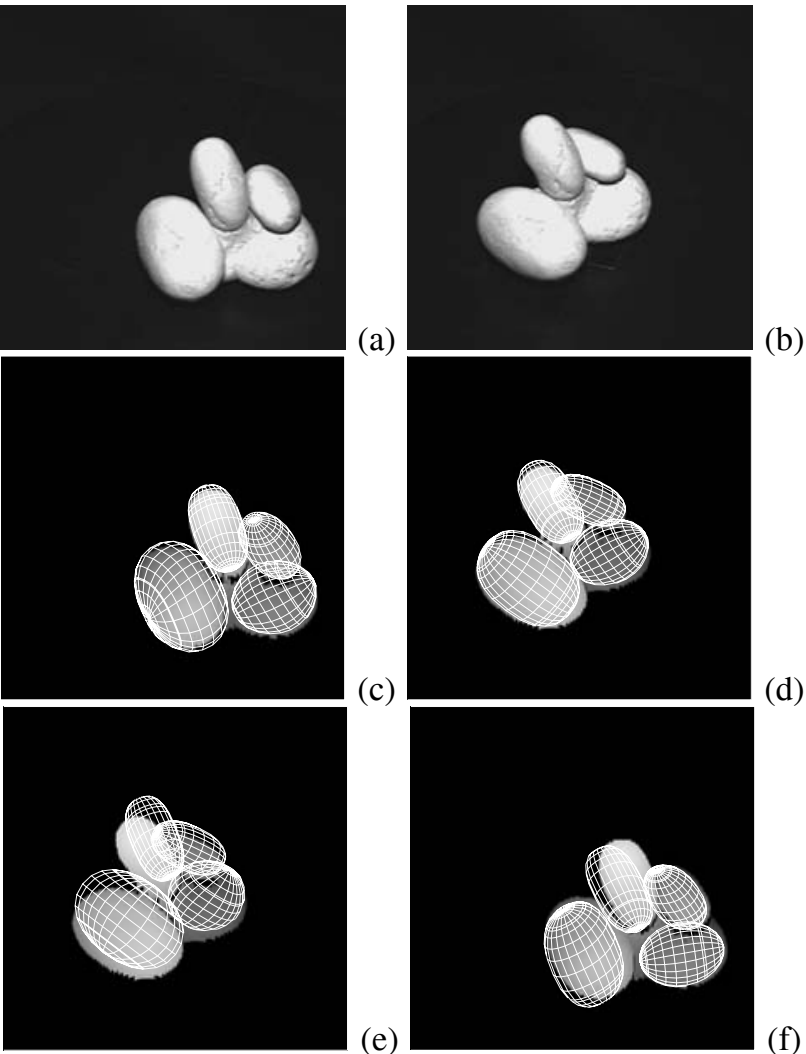

(c)

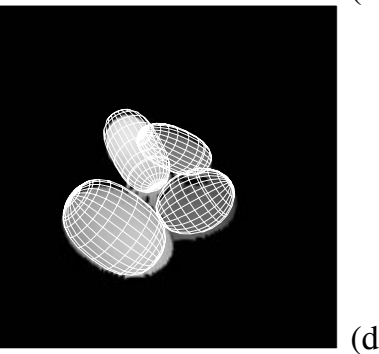

(e)

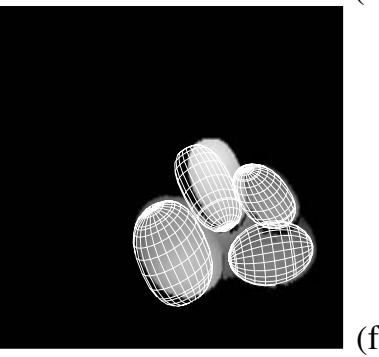

(d)

Fig. 4. Registration of two real range images based on recovered superellipsoids (a) intensity image view1, (b) intensity image view2, (c) range image view1 with recovered superellipsoids (d) range image2 with recovered superellipsoids (e) models recovered from view2 overlaid over range image view1 using the recovered transformation (f) models recovered from view1 overlaid over range image 2 using the recovered transformation. Residual transformation: $\mathbf{t}=(18.21,21.84,-5.91), \mathbf{n}=(-0.72,-0.62,-0.69)$, $\theta=9.6^{\circ}$

were computed based on moments of recovered superellipsoids and another set of estimates based on moments of range image data points where moments of the objects were approximated as sums over $N$ range data points $\left(x_{i}, y_{i}, z_{i}\right)$

$$
m_{p q r}=\sum_{i=1}^{N} x_{i}^{p} y_{i}^{q} z_{i}^{r} .
$$

Ground truth transformations used in generation of synthetic range images were used to compute residual transformations. The results presented in Figs. 5-7 compare precision of estimates computed from moments of recovered models to estimates based on moments of range image data points. Figures marked with (a) and (b) represent range images overlaid with wire frames of recovered superellipsoids of the first and the second view respectively. Figures (c) represent range image of the second view overlaid by the recovered model from the first view using transformation estimate computed from moments of raw range image data points from (60). Similarly, figures (d) show range image of the second view overlaid by the recovered model from the first view using transformation estimate computed from moments of recovered superellipsoid models. Comparison of parameters of residual transformations clearly shows that the method based on moments of recovered superellipsoid models is superior to the method based on 
moments of raw range image data points. On the other hand, the estimate based on moments of recovered superellipsoids is not completely precise since it is not possible to recover a precise superellipsoid model from a single range image.

The error residuals of recovered estimates in case of real and synthetic range images were less than $10^{\circ}$ in rotation and less than $10 \%$ of the object size in translation.
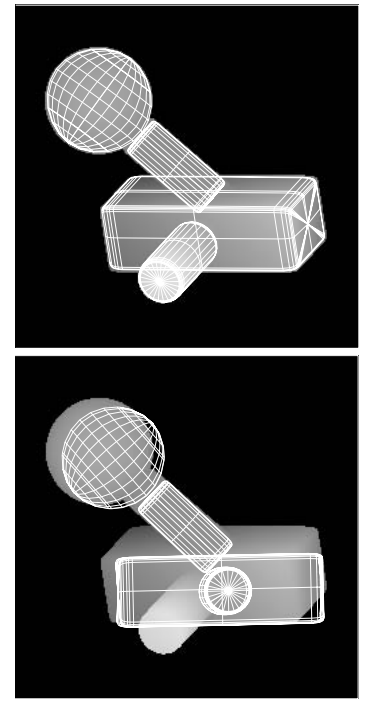

(a)
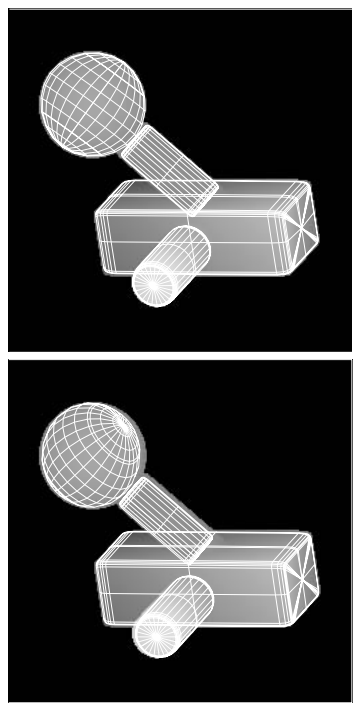

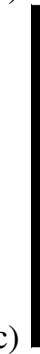

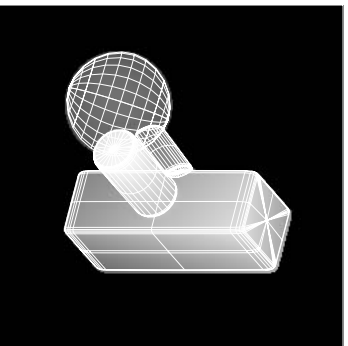

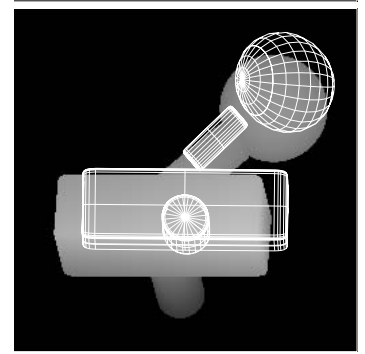

(a)
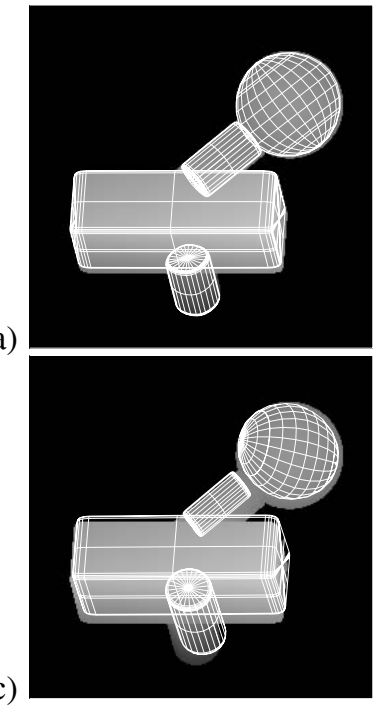

Fig. 7. Comparison of recovered rigid transformations based on moments of recovered models to recovered rigid transformation based on moments of range data points: (a) first view, (b) second view, (c) recovered rigid transformations based on moments of range data, residual transformation $\mathbf{t}=(1.4,-68.1,-3.5), \mathbf{n}=(0.94,0.33,0.04), \theta=21.6^{\circ}$ (d) recovered rigid transformations based on moments of recovered models, residual transformation $\mathbf{t}=(0.1,16.7,-10.4), \mathbf{n}=(0.52,-0.81,0.28), \theta=1.4^{\circ}$.

\section{CONCLUSIONS}

Fig. 5. Comparison of recovered rigid transformations based on moments of recovered models to recovered rigid transformation based on moments of range data points: (a) first view, (b) second view, (c) recovered rigid transformations based on moments of range data, residual transformation $\mathbf{t}=(68.21,71.84,-75.91), \mathbf{n}=(-0.67,-0.74,-0.08), \theta=33.6^{\circ}(\mathrm{d})$ recovered rigid transformations based on moments of recovered models, residual transformation $\mathbf{t}=(0.83,-0.74,-2.17), \mathbf{n}=(-0.19,-0.90,0.38)$, $\theta=0.9^{\circ}$.
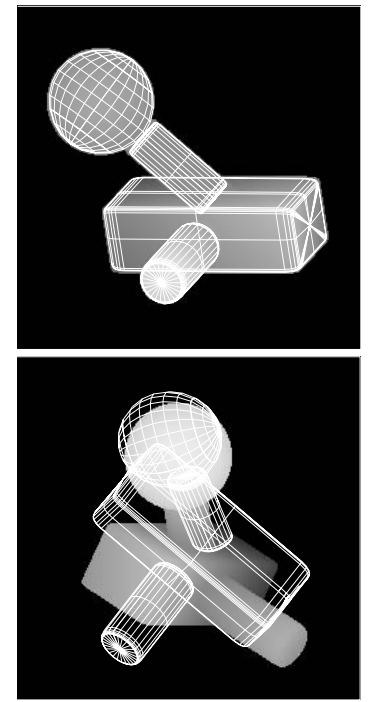

(a)

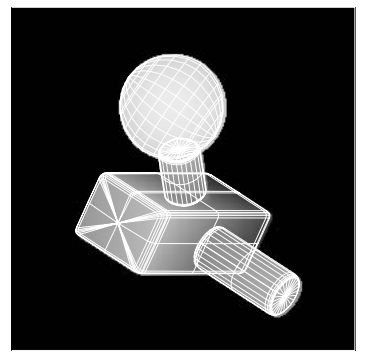

(b)

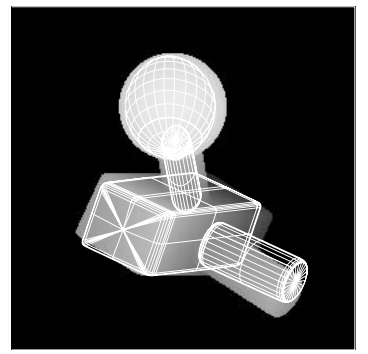

(d)
Fig. 6. Comparison of recovered rigid transformations based on moments of recovered models to recovered rigid transformation based on moments of range data points: (a) first view, (b) second view, (c) recovered rigid transformations based on moments of range data, residual transformation $\mathbf{t}=(101.1,-121.1,120.1), \mathbf{n}=(-0.12,0.74,0.65), \theta=81.6^{\circ}(\mathrm{d})$ recovered rigid transformations based on moments of recovered models, residual transformation $\mathbf{t}=(0.1,16.7,-10.4), \mathbf{n}=(-0.02,0.51,0.86)$, $\theta=8.4^{\circ}$.
We derived closed form expressions for two-dimensional Cartesian moment $m_{p q}$ of order $p+q$ of a superellipse and the three-dimensional Cartesian moment $m_{p q r}$ of order $p+q+r$ of a superellipsoid. These results can be directly used to compute zeroth, first, and second order moments with well known physical meaning as area or volume, center of gravity and moments of inertia as well as to compute higher order moments used in applications of various moment invariants. To demonstrate the correctness of derived expressions, we computed area and moments of inertia for standard twodimensional shapes (rectangle, ellipse, rhomb) and volume and moments of inertia for standard three-dimensional shapes (plate, elliptical cylinder, ellipsoid). We further showed how moments of a transformed object can be computed as linear combinations of moments of the original object if the transformation can be represented with polynomials. Explicit derivations were given for translation, rotation, linear tapering and parabolic bending as well as their combinations.

Feasibility of the proposed registration method based on moments was demonstrated with a registration of two real range views. Experiments with synthetic range images and know ground truth transformation showed significantly better performance of range image registration based on moments of recovered superellipsoid models as compared to registration based on moments of range image data points. This is due to reduced effects of self-occlusion of parts and independence of computed moments on the density of range image data points. The error residuals of recovered estimates were less than $10^{\circ}$ in rotation and less than $10 \%$ of the object size in translation.

The presented results can also be used for object recognition with moments and/or moment invariants as object features. 


\section{APPENDIX I}

\section{BETA AND GAMMA FUnCTIONS}

Beta function is defined as

$$
B(x, y) \equiv 2 \int_{0}^{\pi / 2}(\sin \phi)^{2 x-1}(\cos \phi)^{2 y-1} d \phi
$$

and related to gamma function as follows

$$
B(x, y)=\frac{\Gamma(x) \Gamma(y)}{\Gamma(x+y)} .
$$

For completeness we provide the definition of the gamma function

$$
\Gamma(x)=\int_{0}^{+\infty} t^{x} e^{-t} d t
$$

and the well know equalities for the gamma function used in further derivations

$$
\begin{aligned}
\Gamma(x+1) & =x \Gamma(x) \\
\Gamma(n) & =(n-1) ! \\
\Gamma(1 / 2) & =\sqrt{\pi} .
\end{aligned}
$$

From (64) and (66) it follows that for half integer arguments $(n=1,2,3, \ldots)$

$$
\Gamma(1 / 2+n)=\frac{1 \cdot 3 \cdot 5 \cdots(2 n-1)}{2^{n}} \sqrt{\pi} .
$$

Below we derive the intermediate result frequently used in computing moments of superellipses and superellipsoids

$$
\begin{aligned}
B(x, x+1) & =\frac{\Gamma(x) \Gamma(x+1)}{\Gamma(2 x+1)} \\
& =\frac{x \Gamma(x) \Gamma(x)}{2 x \Gamma(x+1)} \\
& =\frac{1}{2} B(x, x) .
\end{aligned}
$$

Since $\Gamma(x)$ approaches $+\infty$ for $x \rightarrow 0^{+}$and $-\infty$ for $x \rightarrow 0^{-}$ we have to compute the limits for the beta function terms for the case of rectangular shapes, when $\epsilon \rightarrow 0^{+}$

$$
\begin{aligned}
\lim _{\epsilon \rightarrow 0^{+}} \epsilon B(a \epsilon, b \epsilon) & =\lim _{\epsilon \rightarrow 0^{+}} \epsilon \frac{\Gamma(a \epsilon) \Gamma(b \epsilon)}{\Gamma((a+b) \epsilon)} \\
& =\lim _{\epsilon \rightarrow 0^{+}} \epsilon \frac{\frac{\Gamma(a \epsilon+1)}{a \epsilon(b \epsilon+1)}}{\frac{\Gamma((a+b) \epsilon+1)}{(a+b) \epsilon}} \\
& =\left(\frac{1}{a}+\frac{1}{b}\right) \lim _{\epsilon \rightarrow 0^{+}} \frac{\Gamma(a \epsilon+1) \Gamma(b \epsilon+1)}{\Gamma((a+b) \epsilon+1)} \\
& =\frac{1}{a}+\frac{1}{b}
\end{aligned}
$$

and

$$
\begin{aligned}
\lim _{\epsilon \rightarrow 0^{+}} \epsilon B(a \epsilon, b \epsilon+1) & =\lim _{\epsilon \rightarrow 0^{+}} \epsilon \frac{\frac{\Gamma(a \epsilon+1)}{a \epsilon} \Gamma(b \epsilon+1)}{\Gamma((a+b) \epsilon+1)} \\
& =\frac{1}{a} \lim _{\epsilon \rightarrow 0^{+}} \frac{\Gamma(a \epsilon+1) \Gamma(b \epsilon+1)}{\Gamma((a+b) \epsilon+1)} \\
& =\frac{1}{a} .
\end{aligned}
$$

\section{APPENDIX II \\ COMPUTING TRANSFORMATIONS OF MOMENTS With MATHEMATICA}

For the case of transformation where the $f_{x}, f_{y}, f_{z}$ are polynomials with multiple variables $x, y, z$ and the $p, q, r$ of $M_{p q r}$ are fixed, the transformation of moments can be computed symbolically with a program in Mathematica presented in Fig. 8. If the object transformed is not a superellipsoid, the section between the comment lines $(* *)$ should be removed.

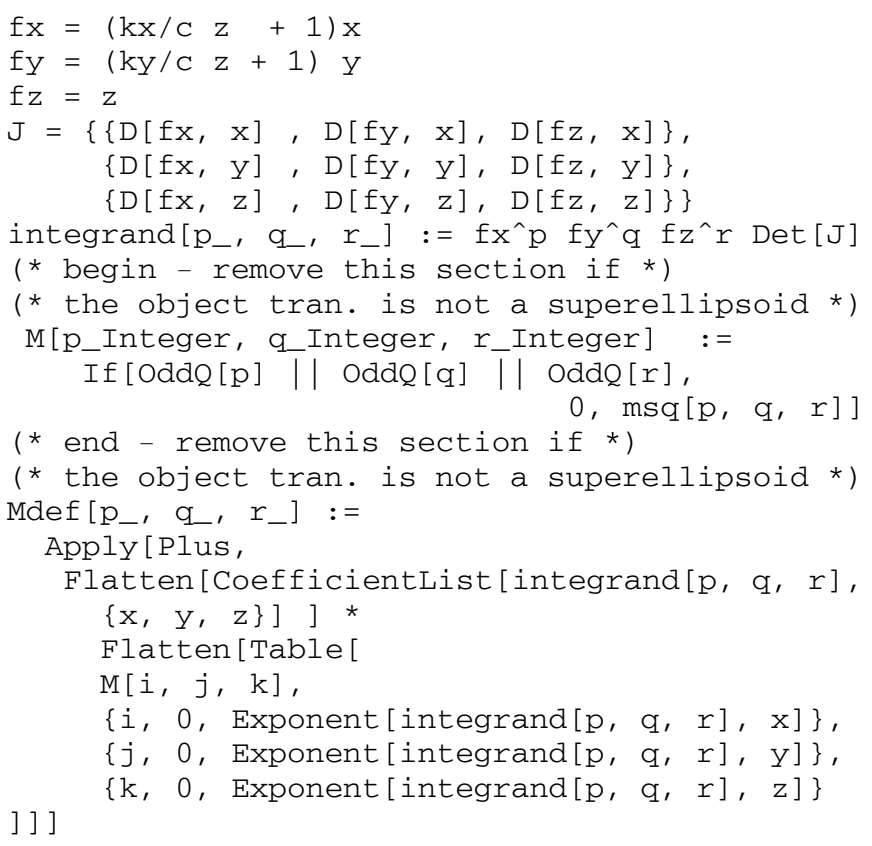

Fig. 8. A program in Mathematica that symbolically computes transformations of moments for the case of superellipsoids.

\section{REFERENCES}

[1] R. J. Prokop and A. P. Reeves, "A survey of moment-based techniques for unoccluded object representation and recognition," Computer Vision, Graphics, and Image Processing. Graphical Models and Image Processing, vol. 54, no. 5, pp. 438-460, 1992.

[2] F. A. Sadjadi and E. L. Hall, "Three-dimensional moment invariants," IEEE Transactions on Pattern Recognition and Machine Intelligence, vol. PAMI-2, no. 2, pp. 127-136, March 1980.

[3] C. Lo and H. Don, "3d moment forms: Their construction and application to object identification and positioning," IEEE Transactions on Pattern Recognition and Machine Intelligence, vol. 11, no. 10, pp. 10531064, October 1989.

[4] A. G. Mamistvalov, "n-dimensional moment invariants and conceptual mathematical theory of recognition n-dimensional solids," IEEE Transactions on Pattern Recognition and Machine Intelligence, vol. 20, no. 8, pp. 819-831, August 1998.

[5] J. M. Galvez and M. Canton, "Normalization and shape recognition of three-dimensional objects by 3D moments," Pattern Recognition, vol. 26, no. 5, pp. 667-681, 1993.

[6] A. Jaklič, A. Leonardis, and F. Solina, Segmentation and Recovery of Superquadrics, ser. Computational imaging and vision. Dordrecth/Boston/London: Kluwer Academic Publishers, 2000, vol. 20. 
[7] M. Y. Zarrugh, "Display and inertia parameters of superellipsoids as generalized constructive solid geometry primitives," in Proceedings of the 1985 ASME International Computers in Engineering Conference and Exhibition, vol. 1, 1985, pp. 317-328.

[8] F. Solina and R. Bajcsy, "Recovery of parametric models from range images: the case for superquadrics with global deformations," IEEE Transactions on Pattern Analysis and Machine Intelligence, vol. 12, no. 2, pp. 131-147, 1990.

[9] P. Whaite and F. P. Ferrie, "From uncertainty to visual exploration," IEEE Transactions on Pattern Analysis and Machine Intelligence, vol. 13, no. 10, pp. 1038-1049, October 1991.

[10] P. J. Besl and N. D. McKay, "A method for registration of 3-D shapes," IEEE Transactions on Pattern Analysis and Machine Intelligence, vol. 14, no. 2, pp. 239-256, February 1992.

[11] G. Turk and M. Levoy, "Zippered polygon meshes from range images," in SIGGRAPH'94 Computer Graphics Proceedings, Annual Conference Series, 1994, pp. 311-247.

[12] G. Blais and M. D. Levine, "Registering multiview range data to create 3D computer objects," IEEE Transactions on Pattern Analysis and Machine Intelligence, vol. 17, no. 8, pp. 820-824, 1995.

[13] C. Dorai, J. Weng, and A. K. Jain, "Optimal registration of object views using range data," IEEE Transactions on Pattern Analysis and Machine Intelligence, vol. 19, no. 10, pp. 1131-1138, 1997.

[14] A. H. Barr, "Superquadrics and angle-preserving transformations," IEEE Computer Graphics and Applications, vol. 1, no. 1, pp. 11-23, January 1981.

[15] F. Solina, "Shape recovery and segmentation with deformable part models," Ph.D. dissertation, University of Pennsylvania, 1987, technical Report MS-CIS-87-111.

[16] A. Leonardis, A. Jaklič, and F. Solina, "Superquadrics for segmentation and modeling range data," IEEE Transactions on Pattern Recognition and Machine Intelligence, vol. 19, no. 11, pp. 1289-1295, November 1997.

[17] K. Arun, T. Huang, and S. Blostein, "Least-squares fitting of two 3$\mathrm{d}$ point sets," IEEE Transactions on Pattern Recognition and Machine Intelligence, vol. 9, no. 5, pp. 698-700, September 1987.

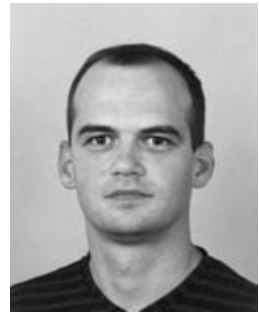

Aleš Jaklič is a teaching assistant at the Faculty of Computer and Information Science, University of Ljubljana He received the B.S. and M.S. degrees in electrical engineering and the Ph.D. degree in computer science from the University of Ljubljana, Slovenia in 1989, 1992, and 1997, respectively. During visits to the University of Pennsylvania in 1990, 1992, 1995, and 1998 he worked as a research assistant at the GRASP Laboratory, University of Pennsylvania and was a post-doctoral Partridge Fellow in 1997 at Fitzwilliam College, University of Cambridge, England. His research interests include range image interpretation, 3D shape reconstruction, multimedia and Internet technologies.

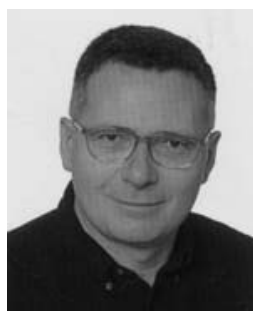

Franc Solina is a professor of computer science at the University of Ljubljana and Head of Computer Vision Laboratory at the Faculty of Computer and Information Science. He received the B.S. and M.S. degree in Electrical Engineering from the University of Ljubljana, Slovenia in 1979 and 1982, respectively, and the Ph.D. degree in computer science from the University of Pennsylvania in 1987. His research interests include range image interpretation, 3D shape reconstruction, panoramic imaging, and applications of computer vision in the arts. 